\title{
Solid-state laser refrigeration at GPa pressures
}

\author{
Abbie G. Ganas, ${ }^{\dagger}$ Elena A. Dobretsova, ${ }^{\ddagger}$ Anupum Pant, $\stackrel{\ddagger}{\ddagger}$ Baptiste Journaux, \\ Xiaojing Xia,,$^{\S}$ R. Greg Felsted, ${ }^{\dagger}$ and Peter J. Pauzauskie*,‡,\| \\ $\dagger$ Department of Chemistry, University of Washington, Seattle, WA 98195, USA \\ $\ddagger$ Department of Materials Science $\mathcal{E}$ Engineering, University of Washington, Seattle, WA \\ 98195, USA \\ \Physical \& Computational Sciences Directorate, Pacific Northwest National Laboratory, \\ Richland, WA 99352, USA \\ $\S$ Molecular Engineering 83 Science Institute, University of Washington, Seattle, WA 98195, \\ USA \\ || Physical \& Computational Sciences Directorate, Pacific Northwest National Laboratory, \\ Richland, WA 99352, USA \\ E-mail: peterpz@uw.edu
}

\begin{abstract}
Although solid-state laser-refrigeration recently has been demonstrated to reach cryogenic temperatures in vacuum, to date the solid-state laser refrigeration of materials at elevated pressure conditions has remained unexplored. Here we demonstrate the laser cooling of ytterbium-doped yttirum-lithium-fluoride $\left(10 \% \mathrm{Yb}^{3+}: \mathrm{YLiF}_{4}\right.$, or $\mathrm{Yb}: \mathrm{YLF})>17 \mathrm{~K}$ below room temperature at pressures $>4 \mathrm{GPa}$ in a diamond anvil cell using lithium fluoride and ice-VII as a quasi-hydrostatic pressure media. Temperature measurements are quantified using a ratiometric-thermometry approach involving a Boltzmann fit to excited states distribution through $4 f$ - $4 f$ Stark-level transitions from the $\mathrm{Yb}^{3+}$ ions that occur between the ${ }^{2} \mathrm{~F}_{5 / 2}$ and ${ }^{2} \mathrm{~F}_{7 / 2}$ manifolds. At pressures
\end{abstract}


between 7 and 12 GPa the YLF grains are observed to undergo a martensitic phase transition from a tetragonal scheelite phase (space group $I 4_{1} / a, \mathrm{Z}=4$, No. 88) to a monoclinic fergusonite phase (space group $I 2 / a, \mathrm{Z}=4$, No. 15 ) which modifies the crystal field splitting of the ground- and excited- state manifolds, but is observed to not eliminate laser cooling. Solid-state laser refrigeration at extreme pressures could allow researchers to use rapid photothermal cycling to explore temperature-dependent properties of materials, including electronic-structure phase-transitions, without the need for external cryostats.

\section{Keywords}

laser refrigeration | diamond anvil cell | high pressure | scheelite | phase transition

\section{Introduction}

Laser heating within a diamond anvil cell (DAC) has led to a range of significant advances towards the understanding of both the fundamental properties ${ }^{1}$ and also the synthesis of materials at extreme conditions. Since Takahashi \& Bassett's original work in the late 1960s using laser heating to synthesize diamond from graphite, ${ }^{2}$ laser heating at extreme conditions has enabled scientists to study matter at conditions relevant to planetary interiors ${ }^{3,4}$ and

also to synthesize a wide variety of new materials with unique phases and compositions. ${ }^{5-7}$ Controlling the temperature within DACs has also led to recent reports of metal-hydride materials with superconducting phase transitions near room temperature. ${ }^{8-10}$

In contrast to laser heating, solid-state laser refrigeration at extreme conditions has remained unexplored. The refrigeration of condensed phases was reported first in 1995 through the use of a metal-fluoride glass that was doped with trivalent ytterbium ions. ${ }^{11}$ During solidstate laser refrigeration, a material is refrigerated through anti-Stokes fluorescence. ${ }^{12}$ A laser, with low optical entropy, excites electronic levels within ytterbium ions which then couple 
to optical phonons provided by the host lattice. This phonon-mediated excited-state upcoversion is followed by the spontaneous emission of high-entropy photoluminescence that transports heat away from the material. Myriad materials have been shown to laser cool in the last two decades. The transition from amorphous glasses to crystalline ceramics such as ytterbium-doped $\mathrm{YLiF}_{4}(\mathrm{Yb}: \mathrm{YLF})$ has had a major impact on the minimum achievable temperatures. Cooling from room temperature to cryogenic temperatures $(\sim 90 \mathrm{~K})$ in vacuum has recently been demonstrated. ${ }^{13}$ More recently, solid-state laser cooling in liquid water has led to the first experimental demonstration of cold Brownian motion ${ }^{14}$ since Einstein's seminal 1905 paper on isothermal Brownian motion. ${ }^{15}$

To date it has not been clear whether solid-state laser refrigeration is possible within a DAC due to the requirement for blue-shifted, anti-Stokes photons to escape and transport heat from the host material. Laser refrigeration within a DAC poses potential challenges for laser cooling considering the high optical index of refraction of diamond $(\mathrm{n}=2.5)$, a large amount of metallic materials within a DAC could prevent the escape of anti-Stokes photoluminescence, and the large conductive heat load, especially when the particles are in contact with the diamond, which has a high thermal conductivity. Additionally, high pressure phase transitions are known to occur for YLF, ${ }^{16,17}$ which can lead to significant changes to the crystal-field (Stark) levels of lanthanide ions. In particular, increasing the splitting of the ground state manifold at high pressures could decrease the efficiency of phonon-mediated upconversion. ${ }^{12}$

Here we report the first experimental demonstration of solid-state laser refrigeration of both individual Yb:YLF microcrystals and bulk Yb:YLF at pressures \& 11 GPa, opening up new experimental possibilities for the rapid photothermal control of the temperatures of matter at extreme conditions. We observe that Yb:YLF in the scheelite phase cools i, 20 $\mathrm{K}$ below room temperature at a pressure of $\sim 4 \mathrm{GPa}$ for both the single crystal and bulk. At pressures above $7 \mathrm{GPa}$ the tetratgonal scheelite phase undergoes a martensitic second order phase transition to a monoclinic fergusonite phase with increased crystal field splitting. 
Despite the phase transition, laser refrigeration \& $5 \mathrm{~K}$ below room temperature is observed for the fergusonite phase for both the single crystal and bulk, opening up a new range of potential applications of laser cooling in high pressure science. Finally, future avenues to optically refrigerate Yb:YLF in the fergusonite phase are discussed.
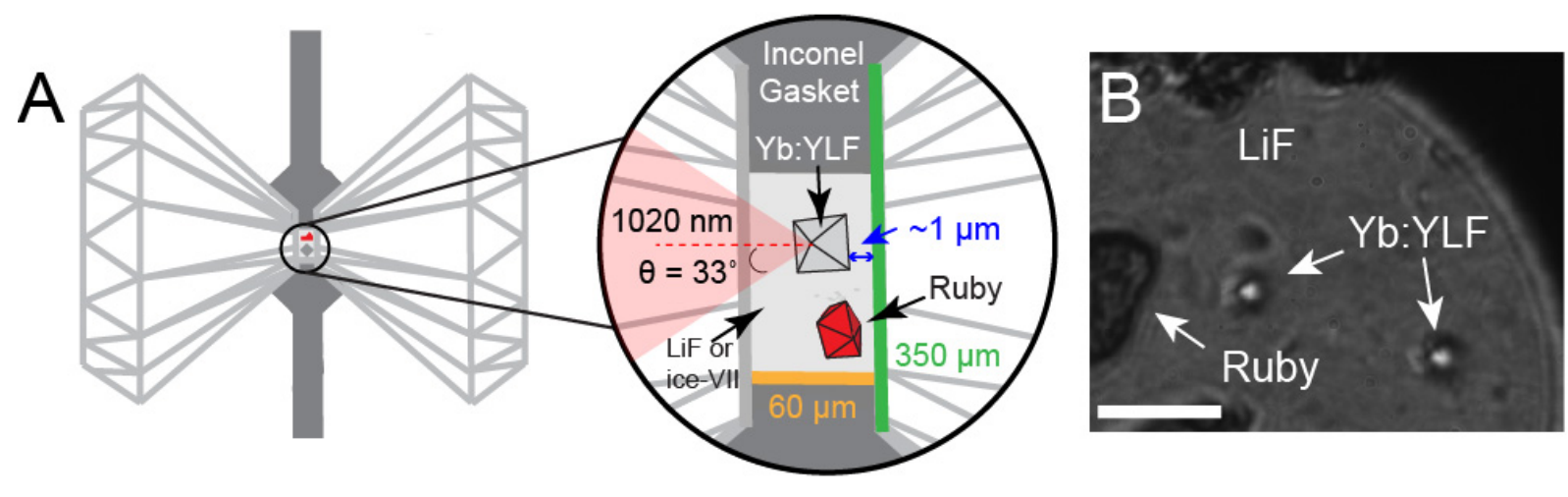

Figure 1: Experimental Schematic (A) An illustration of the diamond anvil cell chamber. Inside the chamber includes ruby, for pressure sensing, Yb:YLF, the sample, and finely ground $\mathrm{LiF}$, the quasi-hydrostatic pressure medium. The critical angle of the microscope objective is denoted as $\theta$. (B) an optical micrograph of the DAC chamber. Components of the chamber are labeled in white. Scale bar is 10 microns.

\section{Results and Discussion}

Merril-Basset and modified Bx90 type DACs were utilized to probe the Yb:YLF samples with increasing pressure (up to $12 \mathrm{GPa}$ ). As seen in Fig 1, LiF serves as the pressure transmitting medium as it maintains a quasi-hydrostatic environment in the desired pressure range of the experiment and is transparent to $1020 \mathrm{~nm}$ light, the main excitation source for this study's experiments. ${ }^{18}$ For one set of experiments, individual YLF micro-grains were first transferred to the anvil culet (Fig. 1A). It is unclear how the YLF grains reoriented upon sealing and pressurization. YLF grains were observed to move in LiF after reaching high pressures. This is illustrated in SI Appendix Fig. S1. It is unlikely that the Yb:YLF 

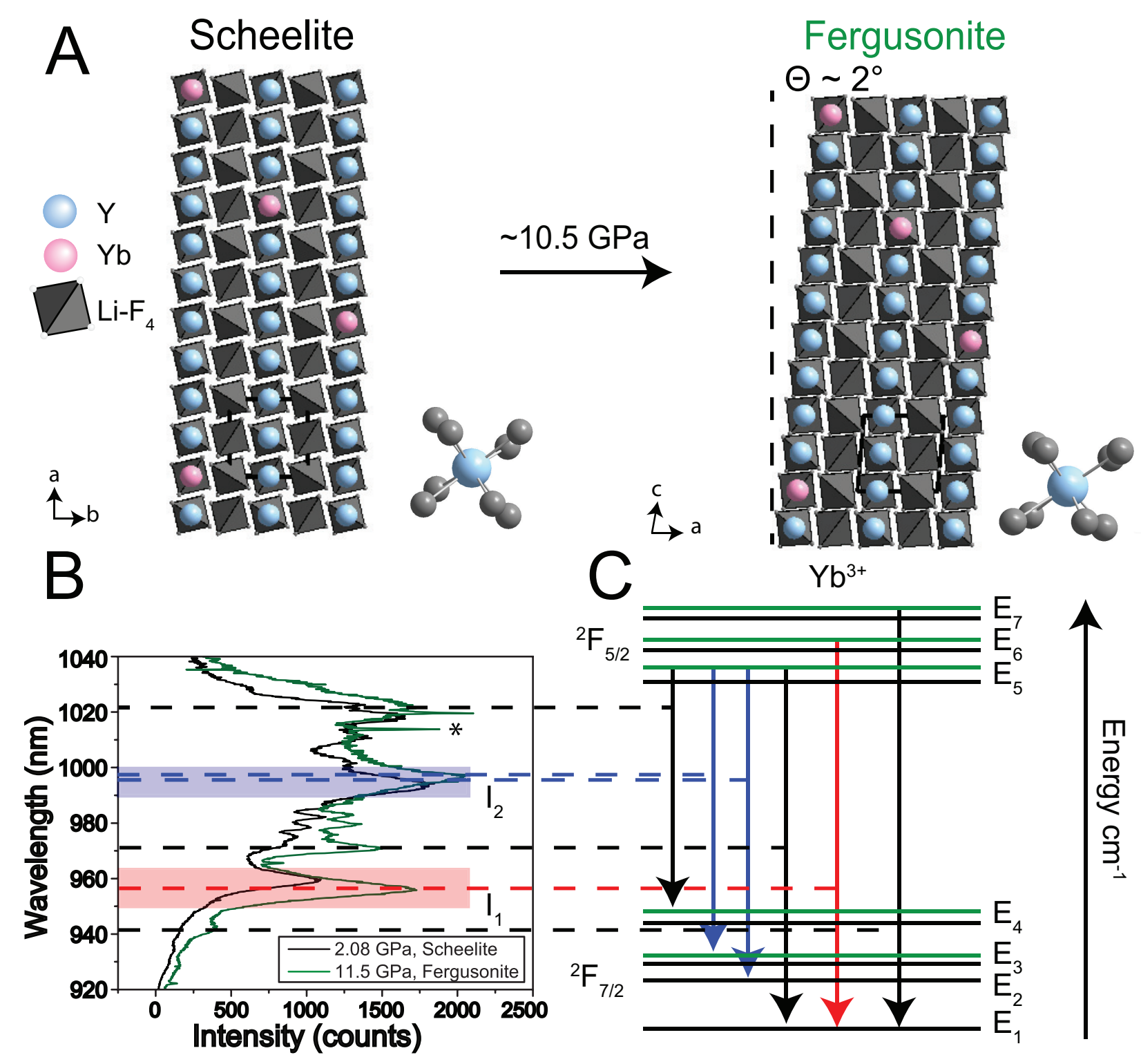

Figure 2: (A) The phase transition of Yb:YLF from tetragonal Scheelite $\left(I 4_{1} / a, \mathrm{Z}=4\right)$ to monoclinic Fergusonite $(I 2 / a, \mathrm{Z}=4)$. The small inset illustrates the local symmetry of the $\mathrm{Y} / \mathrm{Yb}$ ion next to the 8 nearest $\mathrm{F}$ neighbors. The local symmetry is reduced from $\mathrm{S}_{4}$ to $\mathrm{C}_{2}$ when transitioning from scheelite to fergusonite, respectively. (B) The full emission spectrum of $\mathrm{Yb}^{3+}$ ion at elevated pressure. The black line denotes the emission spectrum in the scheelite phase; the green represents the emission spectrum of the fergusonite phase. Key energy transitions are marked with a dashed line. The ${ }^{*}$ denotes a cosmic ray. (C) The Stark manifold of the $\mathrm{Yb}^{3+}$ ion. The key energy transitions denoted in (B) are matched to their respective energy levels in the manifold. The energy transitions in blue and red represent the levels used for Boltzmann ratiometric thermometry analysis. 
grains remained in contact with the diamond culet after sealing as discussed in heat transfer simulations discussed in SI Appendix Fig. S7. Shown in Figure 4B and D is a second set of experiments, which explore the optical refrigeration of approximately half of the DAC chamber filled with Yb:YLF micro-crystals, or a bulk sample set. In both experiments, the excitation laser was focused through the opposite anvil to individual Yb:YLF grains or the bulk sample, as seen in the inset in Fig. 1A. Yttrium lithium fluoride is a uniaxial crystal with polarization-dependent absorption and emission spectra. A partially polarized diode laser was utilized to excite the $\mathrm{Yb}^{3+}$ emission. In order to account for polarization-dependent emission the DAC was returned to its original position and orientation in front of the $50 \mathrm{x}$ objective following every pressure adjustment. The optical micrograph shown in Fig. $1 B$ illustrates the experimental chamber at the highest pressure of $11.5 \mathrm{GPa}$. As the pressure was increased, ruby was utilized to monitor not only the pressure, but the hydrostaticity of the DAC chamber, following procedures found in Chai and Brown (1996) et al. ${ }^{19}$ and seen in SI Appendix Fig. S3. By measuring the splitting distance of the $\mathrm{R}_{1}$ and $\mathrm{R}_{2}$ luminescence peaks as the pressure is increased or decreased, it was determined that the DAC chamber was hydrostatic over the pressure ranges investigated in this study. Therefore, any changes in photoluminescence spectra collected are due to compression only, not tensile or sheer stresses due to a nonhydrostatic environment.

The local environment of the scheelite Yb:YLF crystal contains a trivalent yttrium/ytterbium cation and a monovalent lithium cation coordinated by eight fluoride and 4 fluoride anions, respectively, as seen in Fig. $2 A$. Under ambient conditions, the quasi-tetrahedral $\mathrm{LiF}_{4}{ }^{3-}$ subunit has been characterized to have ionic bonding, which is more easily compressible than the $\mathrm{Y} / \mathrm{Yb}-\mathrm{F}$ bond. ${ }^{20-23}$ The point-group symmetry of the trivalent cation site is $\mathrm{S}_{4}$. According to experimental ${ }^{16,17}$ and theoretical literature,${ }^{24-26}$ as a YLF crystal undergoes compression in a hydrostatic environment, its phase changes from a scheelite phase (tetragonal, spacegroup $I 4_{1} / a$ ) to a fergusonite phase (monoclinic, spacegroup $I 2 / a$ ), as seen in Fig. $2 A$. The local symmetry of the trivalent cation site reduces to $\mathrm{C}_{2}$ after the transition to the fergusonite 
phase. Raman and XRD pressure measurements ${ }^{16,17}$ place this phase transition pressure in a wide range pressures. Optical luminescence measurements of rare earth ions substitutionally doped into the yttirum site ${ }^{16}$ have observed subtle reductions of local point-group symmetry at pressures above $10 \mathrm{GPa}$. Discontinuities in the phonon frequencies during Raman measurements, due to the stiffening of the $\mathrm{LiF}_{4}{ }^{3-}$ bonds, indicated a structural phase transformation at $7 \mathrm{GPa} .{ }^{21}$ As previous papers have shown that photoluminescence from $\mathrm{Nd}$ and $\mathrm{Eu}$ ions can detect the transition from scheelite to fergusonite, we relied on these measurements for $\mathrm{Yb}$, and the well known phase transition pressure of $i, 10 \mathrm{GPa}$, to track the phase transition of our YLF crystals. Overall, the phase transformation from scheelite to fergusonite is slow with the major structural changes occurring at ; 10 GPa. All studies showed a reversible transformation from the fergusonite phase back to the scheelite phase when going back to ambient pressures, which is corroborated by our measurements. Theoretical calculations have predicted these phase transitions can occur at a range of pressures between $9^{27}$ and $5.5^{24} \mathrm{GPa}$.

Figure $2 B$ illustrates the fluorescence spectrum of a trivalent $\mathrm{Yb}^{3+}$ dopant in the YLF host crystal. Based on the crystal field interactions from the host lattice, the ${ }^{2} \mathrm{~F}_{7 / 2}$ (ground state) and ${ }^{2} \mathrm{~F}_{5 / 2}$ (excited state) spin-orbit manifolds are further split into four and three Stark levels, respectively, giving a total of 12 possible transitions, as seen in Fig. 2C. The fluorescence peaks and their corresponding vibronic sidebands have been characterized by previous studies at low temperature and ambient pressure. ${ }^{28,29}$ From these studies, the energy transitions were derived based on the $\mathrm{S}_{4}$ point group symmetry's reduced matrix elements and utilized to make predictions on the value of each energy level in the ground and excited state manifolds seen in Fig. $2 C \cdot{ }^{29,30}$ To the best of the authors' knowledge the $\mathrm{Yb}^{3+}$ fluorescence spectrum in YLF has not been investigated while under compressive strain.

Increasing the pressure with the DAC is observed to have a significant impact on the crystal field levels for $\mathrm{Yb}$ ions in YLF. The black line in the emission spectrum of Fig.2B represents the emission when the scheelite phase is at elevated pressure (2.38 GPa) and 


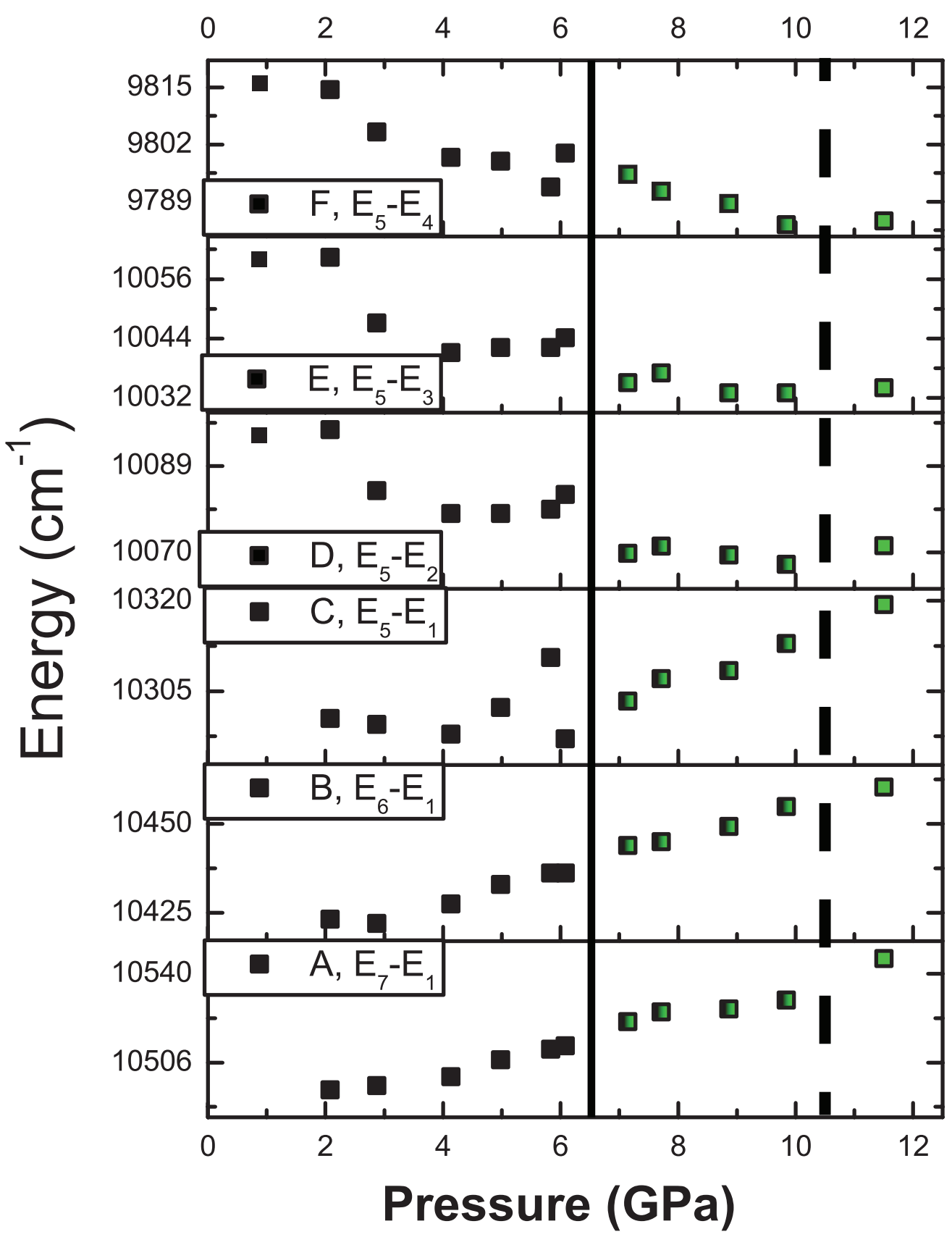

Figure 3: The key energy transitions highlighted in Fig. 2 over all experimental pressures. The green squares and green-black gradient squares indicate the energy transitions in the fergusonite phase and the slow conversion to the fergusonite phase, respectively. The solid line denotes the pressure $(6.5 \mathrm{GPa})$ of observed, consistent discontinuities in the trend-line usually marking a phase transition. This occurs at a much lower pressure than what is previously reported in literature $(10.5 \mathrm{GPa})$, which is denoted with the dashed line. (A) $\mathrm{E}_{7}-\mathrm{E}_{1}$ (B) $\mathrm{E}_{6}-\mathrm{E}_{1}$ (c) $\mathrm{E}_{5}-\mathrm{E}_{1}$ (D) $\mathrm{E}_{5}-\mathrm{E}_{2}$ (E) $\mathrm{E}_{5}-\mathrm{E}_{3}$ (F) $\mathrm{E}_{5}-\mathrm{E}_{4}$ Energy levels in the ${ }^{2} \mathrm{~F}_{7 / 2}$ ground state and the ${ }^{2} \mathrm{~F}_{5 / 2}$ excited state were measured and fit via Levenberg-Marquadt non linear least squares fitting. 
the green line represents when YLF has already transitioned to the fergusonite phase (11.5 GPa). Overall, changes between the PL emission spectra clearly illustrate that the crystal field is being modified by the increasing strain. For example, three of the highlighted energy transitions shift in wavelength: the $\mathrm{E}_{6}-\mathrm{E}_{1}$ transition, the $\mathrm{E}_{5}-\mathrm{E}_{2,3}$ transition, and the $\mathrm{E}_{5}-\mathrm{E}_{4}$ transition gradually blue shift, red shift, and red shift, respectively. The $\mathrm{E}_{5}-\mathrm{E}_{1}$ transition, increases in intensity as the pressure is increased. The $\mathrm{E}_{7}-\mathrm{E}_{1}$ transition both blue shifts and increases in intensity. At a pressure of around $6 \mathrm{GPa}$, a prominent new luminescence peak at a wavelength of $\sim 970 \mathrm{~nm}$ is observed as shown in Fig. 2B, indicating the preliminary onset of the martensitic phase transition. Previous studies ${ }^{31}$ have linked the point group symmetry of a rare earth doped fluoride scheelite with its ligand field Hamiltonian to calculate its vibronic intensity parameters. Modification of the rare earth ion's point group symmetry with pressure can alter its surrounding ligand field and thus alter the intensities of is electronic and vibronic transitions, as can be seen in the $\mathrm{E}_{5}-\mathrm{E}_{1}$ transition $(\lambda \approx 970)$ in Fig.2B.

Figure 3 depicts the change in energy of the key transitions from Fig. $2 B$ over all the pressure points investigated in this study. The emission spectra were fit with a LevenbergMarquadt nonlinear least squares fitting in Python. With the code, the fluorescence peaks were fit to within a $0.1 \%$ error based upon the fluorescence peaks at ambient conditions from Ref. ${ }^{28}$ As the spectra were fit at increasing pressures, the absolute energies of each level in the ${ }^{2} \mathrm{~F}_{7 / 2}$ and ${ }^{2} \mathrm{~F}_{5 / 2}$ manifolds were calculated based on the fitted energy transitions. These calculated energies were then checked with the $\mathrm{E}_{7}-\mathrm{E}_{1}$ energy transition, even as it blue shifted with increasing pressure. Each absolute energy level, from $\mathrm{E}_{1}$ through $\mathrm{E}_{7}$, must add up to the total energy of the $E_{7}-E_{1}$ energy transition of the emission spectrum.

The trends in Fig. 3 of each key energy transition depict discontinuities at approximately $6 \mathrm{GPa}$, which normally indicates a phase transition. To reiterate, literature points to the phase transition to fergusonite at $10.5 \mathrm{GPa}$, but fluorescence experiments utilizing other RE ions show subtle structural changes as low as 5.5 GPa. The discontinuities depicted in 
Fig. 3 for each energy transition therefore illustrate similar trends. Each spectrum was fit with a linear trend-line to measure the energy shift change with increasing pressure of the transitions, as seen in SI Appendix Fig. S5. The most striking of the energy transition trends shown in Fig. 3, the $\mathrm{E}_{7}-\mathrm{E}_{1}$, the $\mathrm{E}_{6}-\mathrm{E}_{1}$, and the $\mathrm{E}_{5}-\mathrm{E}_{1}$, illustrate an increasing energy as the pressure is increased with a slope of $5.3 \pm 0.3 \mathrm{~cm}^{-1} \mathrm{GPa}^{-1}, 4.3 \pm 0.2 \mathrm{~cm}^{-1} \mathrm{GPa}^{-1}$, and 2.0 $\pm 0.4 \mathrm{~cm}^{-1} \mathrm{GPa}^{-1}$, respectively. Even though the individual $\mathrm{E}_{5}-\mathrm{E}_{4}$ transition demonstrates a clear red shift in energy, with a slope of $-2.9 \pm 0.4 \mathrm{~cm}^{-1} \mathrm{GPa}^{-1}$, overall the energy levels in the manifold are increasing, as depicted in Fig. 2C. Subtle structural changes to the YLF host crystal, due to the increasing pressure, reduce the local symmetry of the $\mathrm{Yb}$ ion bonded to the $\mathrm{LiF}_{4}$ tetrahedron to $\mathrm{C}_{2}$. Consequently, the fluorescence peaks convey very gradual shifting, either blue or red shifting, of the energy transitions as the $\mathrm{Yb}$ ion is responding to the changing crystal field.

Figure 4 demonstrates the first measurements of solid-state laser refrigeration for the scheelite phase of YLF at various elevated pressure conditions for both the single crystal and bulk experiments. Ratiometric luminescence thermometry was utilized to measure the temperature trend of a Yb:YLF crystal inside a diamond anvil cell chamber based on the Boltzmann distribution of thermally accessible states. Two fluorescence peaks are chosen for integration, the $\mathrm{E}_{6}-\mathrm{E}_{1}$ and $\mathrm{E}_{5}-\mathrm{E}_{2,3}$ transitions, as seen in the blue and red shaded regions in Fig. 2B. When the laser power is varied, it is observed that the integrated photoluminescence intensities of these two peaks change based on the internal temperature of the host crystal. As the internal temperature of the host crystal decreases, so does the probability of $\mathrm{Yb}$-ions accessing higher energy configurations, which is reflected in the photoluminescence intensity. Under these circumstances, it is expected that the integrated ratio between the aforementioned emission transitions will also decrease. This trend is illustrated for the scheelite phase in Fig. $4 A \& B$. After temperature calibration, the decreasing ratio between the two peaks could be used to quantify the temperature. A temperature decrease of approximately $20 \pm 5 \mathrm{~K}$ below room temperature was determined for the single YLF particle in the 

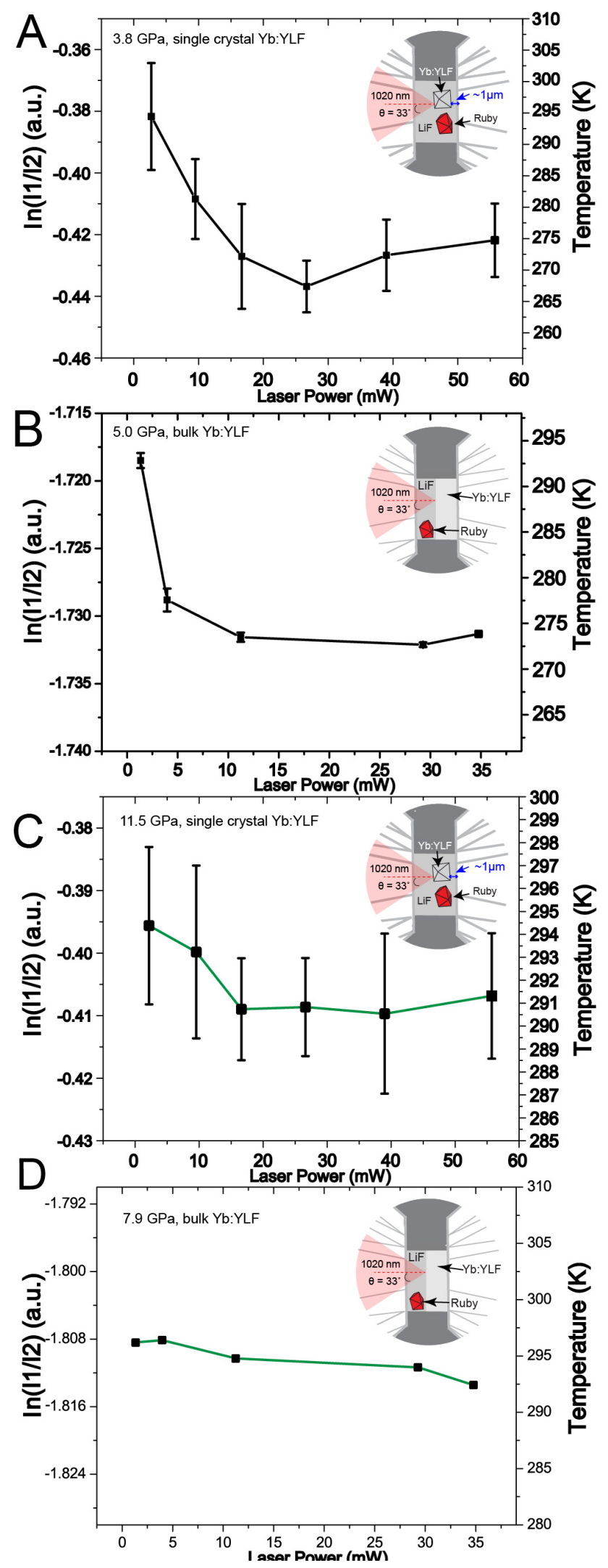

Figure 4: Optical refrigeration data from a Yb:YLF particle in the (A) scheelite and (B) fergusonite phase. Error bars represent the standard deviation of six separate measurements at each laser power. 
scheelite phase at $3.8 \mathrm{GPa}$, as seen in Fig.4A. The magnitude of this temperature change is consistent with numerical finite element simulations shown in SI Appendix Fig. S7. Figure $4 B$ also demonstrates the potential to refrigerate the bulk scheelite phase, as the ensemble was observed to cool approximately $17.0 \pm 0.1 \mathrm{~K}$ below room temperature at a pressure of 5.0 GPa, immediately preceding the onset of the martensitic phase transition. One potential explanation for the smaller net cooling of the bulk sample is re-absorption from adjacent grains of Yb:YLF.

As discussed above, many of $\mathrm{Yb}^{3+}$ 's Stark manifold energy levels are modified with pressure. In particular, the $\mathrm{E}_{5}-\mathrm{E}_{4}$ energy level red shifts as it enters into the fergusonite phase. This has major implications on the net cooling of Yb:YLF as the incident excitation of $1020 \mathrm{~nm}$ is no longer exactly resonant to this transition. Because the incident source is more energetic, anti-Stokes fluorescence still occurs, however, a reduction in the magnitude of cooling is observed, as seen in Fig. $4 C \& D$. For both the single crystal and bulk sample of Yb:YLF, the calibrated magnitude of cooling is much lower for the fergusonite phase than for the scheelite phase, with the former cooling by approximately $4 \mathrm{~K}$ and the latter cooling by approximately $1.96 \pm 0.02 \mathrm{~K}$. As what was seen with the scheelite phase, there is a lower net cooling between the single crystal and the bulk sample. In future experiments, to verfiy this hypothesis, a tunable laser could be utilized to change the incident excitations source's wavelength to match the $\mathrm{E}_{5}-\mathrm{E}_{4}$ energy level as it changes with pressure.

In conclusion, we have demonstrated solid state optical refrigeration for the first time at extreme pressure conditions. Both single micro-crystals and bulk Yb:YLF were placed inside a diamond anvil cell with $\mathrm{LiF}$ as a pressure medium. A martensitic second order phase transition of the Yb:YLF between scheelite and fergusonite phases was observed via photoluminescence spectra from $\mathrm{Yb}^{3+}$ ions. A decrease in the temperature of $20 \pm 5 \mathrm{~K}$ below room temperature was observed for the scheelite phase for a single micro-crystal. Preliminary studies of temperature calibrated single Yb:YLF particles in water demonstrate cooling of approximately $16 \pm 5 \mathrm{~K}$ below room temperature in the scheelite phase at elevated 
pressure (SI Appendix Fig. S6). Raman measurements (SI Appendix Fig. S6B) of the pressure transmitting medium, water, confirm its phase transformation to ice VII. This result illustrates the versatility of the particles cooling a variety of local environments and can potentially lead to achieving localized and fast cooling in-situ in the DAC. Specifically, utilizing these laser cooling capable particles could allow researchers to explore new territories of nucleation/crystallization and chemical fractionation kinetics at high pressures with various materials like high pressure ices that have major planetary applications. ${ }^{32,33}$

\section{Methods}

\section{YLF Synthesis}

All reagents were purchased from Sigma Aldrich and utilized without further purification. The hydrothermal synthesis used to synthesize Yb:YLF nanocrystals was taken from Roder et al with some modifications. ${ }^{14}$ Approximately $0.585 \mathrm{~g}$ (2 mmol, analytical grade) EDTA was dissolved in $10 \mathrm{~mL}$ Millipore DI water and $0.28 \mathrm{~g} \mathrm{LiOH}$, and heated to $80^{\circ} \mathrm{C}$ while stirring. After the EDTA dissolved, $1.8 \mathrm{~mL}$ of $1.0 \mathrm{M}$ (1.8 mmol, 99.999 purity) Yttrium chloride hexahydrate $\left(\mathrm{YCl}_{3} * 6 \mathrm{H}_{2} \mathrm{O}\right)$ and $0.2 \mathrm{~mL}$ of $1.0 \mathrm{M}(0.2 \mathrm{mmol})$ Ytterbium chloride hexahydrate $\left(\mathrm{YbCl}_{3} * 6 \mathrm{H}_{2} \mathrm{O}\right)$ was added to the solution with continuous stirring. This mixture was denoted as solution A. In a separate container, denoted solution B, $0.105 \mathrm{~g}$ (4mmol, analytical grade) $\mathrm{LiF}$ and $0.34 \mathrm{~g}$ (8mmol, analytical grade) of $\mathrm{NH}_{4} \mathrm{HF}_{2}$ were dissolved in 5 $\mathrm{mL}$ of Millipore DI water and heated to approximately $70^{\circ} \mathrm{C}$ with stirring for 1 hour. After the hour, solution B was added dropwise to A with continuous stirring to form a homogeneous white suspension. After 30 minutes, the combined mixture was then transferred to a $23-\mathrm{mL}$ Teflon-lined autoclave (Parr 4747 Nickel Autoclave Teflon liner assembly) and heated to $180^{\circ} \mathrm{C}$ in a Thermo Scientific Heratherm General Protocol Oven (65 L) for 72 hours. After, the autoclave was taken out of the oven and allowed to naturally come to room temperature. The product, Yb:YLF, was sonicated and centrifuged at $4000 \mathrm{rpm}$ with ethanol and Millipore 
DI water three times each. The final white powder was then dried in the oven at $60^{\circ} \mathrm{C}$ for 12 hours followed by calcination in at $300^{\circ} \mathrm{C}$ for 2 hours using a ThermoScientific Lindberg/Blue M tube furnace.

\section{Diamond Anvil Cell Preparation}

A Merrill-Basset and modified Bx90 ${ }^{34}$ diamond anvil cell (DAC) were employed for spectroscopic experiments. Standard, type 1a diamonds with a culet size of $0.350 \mathrm{~mm}$ served as the anvils, which indented an Inconel 718 gasket to roughly $100 \mathrm{um}$ in thickness. A hole was drilled in the center of this indentation with a $0.155 \mathrm{~mm}$ copper wire using electric discharge machining (EDM). Annealed ruby chips and crystals of Yb:YLF were placed into the homemade chamber using a Marzhauser Wetzlar, type MCL-3, nanomanipulator equipped with a tungsten needle. Yb:YLF samples were placed directly in contact with the bottom culet. Ground lithium fluoride was poured into the chamber to serve as the hydrostatic pressure medium. For experiments where the pressure transmitting medium was water, the DAC was submerged in DI water. For both experimental designs, it is unclear whether the Yb:YLF samples moved or reoriented themselves upon sealing and pressurizing the chamber.Utilizing the anvil culets as a Fabry-Perot interferometer, see SI Appendix Fig. S4 for more details, it was determined that at the sealing pressure, $2.38 \mathrm{GPa}$, the gasket thickness was approximately 100 microns in diameter.

Figure 4 exhibits a stark difference in error bars. The reduction in error in Fig.4B and Fig.4D arise from the different detectors used to collect emission signal (See SI Appendix for precise experimental details).

\section{Spectroscopic Measurements}

Spectroscopic measurements were collected with a home built optical set up (see SI Appendix for precise experimental details). The sample chamber was aligned to a $532 \mathrm{~nm}$ Coherent laser and a custom made QPhotonics $1020 \mathrm{~nm}$ diode laser. The $532 \mathrm{~nm}$ laser was utilized 
to determine the pressure of the strain affected ${ }^{2} \mathrm{~T}_{1} \Rightarrow{ }^{4} \mathrm{~A}_{2} \mathrm{Cr}^{3+}$ ruby $\mathrm{R} 1$ line. The $1020 \mathrm{~nm}$ laser was used to excite the $\mathrm{Yb}^{3+}$ photoluminescence.

Raman measurements (See SI Appendix for precise experimental details) were collected with a Renishaw inVia system equipped with a Leica DMIRBE inverted optical microscope. A confocal high end $785 \mathrm{~nm}$ served as the excitation source.

\section{Temperature Calibration}

Cooling down to $250 \mathrm{~K}$ was achieved with a cryostat apparatus using Thermoelectric Coolers (TEC), modified from the initial design by Noguchi \& Okuchi. ${ }^{35}$

\section{Data Availability}

We have provided all relevant data in the text, figures, and SI Appendix.

\section{Acknowledgement}

A.S.G. and P.P. acknowledge primary support for this work from the National Science Foundation (CAREER Award \#1555007, MRSEC ). E.A.D. and A.S.G. also acknowledge support from the National Science Foundation and the UW Molecular Engineering Materials Center, a Materials Research Science and Engineering Center (DMR-1719797). BJ acknowledges the support from by the NASA Solar System Workings Grant 80NSSC17K0775, and by the Icy Worlds, and Habitability of Hydrocarbon Worlds: Titan and Beyond nodes of NASA's Astrobiology Institute (08-NAI5-0021 and 17-NAI8-2-017). The DAC TEC cryostat was developed by B. J. at the University of Washington with the support of the NASA Astrobiology Institute's Virtual Planetary Laboratory, supported by the National Aeronautics and Space Administration through the NASA Astrobiology Institute under solicitation NNH12ZDA002C and Cooperative Agreement Number NNA13AA93A. X.X., A.P., and P.P. acknowledge support from the MURI:MARBLe project under the auspices of the Air Force 
Office of Scientific Research (Award \#FA9550-16-1-0362). The authors acknowledge the insight and fruitful discussions with E. Abramson about many of the experimental aspect of this work. The authors acknowledge Stuart Frazier Diwash Dhakal for assistance with YLF synthesis and Raman spectroscopy, respectively. All authors acknowledge support form the UW Nano-engineered Systems Institute. Part of this work was conducted at the Molecular Analysis Facility, a National Nanotechnology Coordinated Infrastructure site at the University of Washington which is supported in part by the National Science Foundation (grant NNCI-1542101), the University of Washington, the Molecular Engineering \& Sciences Institute, and the Clean Energy Institute. G.F. and P.P. acknowledge support from the ONR-BRC (BAA Announcement N00014-17-S-BA13). The authors acknowledge Stuart Frazier \& Diwash Dhakal for assistance with YLF synthesis and Raman spectroscopy, respectively.

\section{Supporting Information Available}

Supporting Information available.

\section{References}

(1) Drickamer, H. Forty years of pressure tuning spectroscopy. Annu. Rev. Mater. Sci. 1990, 20, 1-18.

(2) Bassett, W. A. The birth and development of laser heating in diamond anvil cells. Rev. Sci. Instrum. 2001, 72, 1270-1272.

(3) Bassett, W. A.; Ming, L.-C. Disproportionation of $\mathrm{Fe}_{2} \mathrm{SiO}_{4}$ to $2 \mathrm{FeO}^{+} \mathrm{SiO}_{2}$ at pressures up to 250 kbar and temperatures up to 3000 C. Phys. Earth Planet. Inter. 1972, 6, $154-160$. 
(4) Millot, M.; Hamel, S.; Rygg, J. R.; Celliers, P. M.; Collins, G. W.; Coppari, F.; Fratanduono, D. E.; Jeanloz, R.; Swift, D. C.; Eggert, J. H. Experimental evidence for superionic water ice using shock compression. Nat. Phys. 2018, 14, 297.

(5) Walsh, J. P. S.; Freedman, D. E. High-Pressure Synthesis: A New Frontier in the Search for Next-Generation Intermetallic Compounds. Acc. Chem. Res. 2018, 51, 1315-1323, PMID: 29812893.

(6) Guerette, M.; Ward, M. D.; Lokshin, K. A.; Wong, A. T.; Zhang, H.; Stefanoski, S.; Kurakevych, O.; Le Godec, Y.; Juhl, S. J.; Alem, N.; Fei, Y.; Strobel, T. A. Synthesis and Properties of Single-Crystalline Na4Si24. Crystal Growth \& Design 2018, 18, 74107418.

(7) Crane, M. J.; Petrone, A.; Beck, R. A.; Lim, M. B.; Zhou, X.; Li, X.; Stroud, R. M.; Pauzauskie, P. J. High-pressure, high-temperature molecular doping of nanodiamond. Sci. Adv 2019, 5 .

(8) Ashcroft, N. W. Hydrogen Dominant Metallic Alloys: High Temperature Superconductors? Phys. Rev. Lett. 2004, 92, 187002.

(9) Drozdov, A.; Kong, P.; Minkov, V.; Besedin, S.; Kuzovnikov, M.; Mozaffari, S.; Balicas, L.; Balakirev, F.; Graf, D.; Prakapenka, V., et al. Superconductivity at 250 K in lanthanum hydride under high pressures. Nature 2019, 569, 528.

(10) Somayazulu, M.; Ahart, M.; Mishra, A. K.; Geballe, Z. M.; Baldini, M.; Meng, Y.; Struzhkin, V. V.; Hemley, R. J. Evidence for Superconductivity above 260 K in Lanthanum Superhydride at Megabar Pressures. Phys. Rev. Lett. 2019, 122, 027001.

(11) Epstein, R.; Buchwald, M.; Edwards, B.; Gosnell, T.; Mungan, C. Observation of laserinduced fluorescent cooling of a solid. Nature 1995, 377, 500-503. 
(12) Seletskiy, D. V.; Melgaard, S. D.; Bigotta, S.; Di Lieto, A.; Tonelli, M.; Sheik-Bahae, M. Laser cooling of solids to cryogenic temperatures. Nat. Photonics 2010, 4, 161-164.

(13) Melgaard, S.; Seletskiy, D.; Di Lieto, A.; Tonelli, M.; Sheik-Bahae, M. Optical refrigeration to $119 \mathrm{k}$, below national institute of standards and technology cryogenic temperature. Opt. Lett. 2013, 38, 1588-1590.

(14) Roder, P.; Smith, B.; Xuezhe, Z.; Crane, M.; Pauzauskie, P. Laser refrigeration of hydrothermal nanocrystals in physiological media. Proc. Natl. Acad. Sci. 2015, 112, $15024-15029$.

(15) Einstein, A. Über die von der molekularkinetischen Theorie der Wärme geforderte Bewegung von in ruhenden Flüssigkeiten suspendierten Teilchen. Annalen der Physik 1905, 322, 549-560.

(16) Shenxin, L.; Yuanbin, C.; Xuyi, Z.; Lizhong, W. High pressure luminescence and pressure induced phase transition for $\mathrm{LiYF}_{4}: \mathrm{Eu}$. J. Alloys Compd. 1997, 255, $1-4$.

(17) Grzechnik, A.; Syassen, K.; Loa, I.; Hanfland, M.; Gesland, J. Y. Scheelite to fergusonite phase transition in $\mathrm{YLiF}_{4}$ at high pressures. Phys. Rev. B 2002, 65, 104102.

(18) Jia-Zeng, G.; Ye, W.; Feng, Z.; Xiang, W.; Ke, Y.; Q, S. Quasi-hydrostatic Limit of LiF as a Pressure Transmitting Medium and Its Equation of States. Chin. Phys. Lett. 2014, 31, 05621.

(19) Chai, M.; Brown, M. Effects of static non-hydrostatic stress on the R lines of ruby single crystals. Geophys. Res. Lett. 1996, 23, 3539-3542.

(20) Miller, S. A.; Rast, H. E.; Caspers, H. H. Lattice Vibrations of LiYF 4 . J. Chem. Phys. 1970, 52, 4172-4175.

(21) Salaün, S.; Fornoni, M. T.; Bulou, A.; Rousseau, M.; Simon, P.; Gesland, J. Y. Lattice 
dynamics of fluoride scheelites: I. Raman and infrared study of and (and Yb). J. Phys.: Condens. Matter 1997, 9, 6941-6956.

(22) Salaün, S.; Bulou, A.; Rousseau, M.; Hennion, B.; Gesland, J. Y. Lattice dynamics of fluoride scheelites: II. Inelastic neutron scattering in and modelization. J. Phys.: Condens. Matter 1997, 9, 6957-6968.

(23) Sen, A.; Chaplot, S. L.; Mittal, R. Rigid ion model of lattice dynamics in the laser host fluoroscheelites $\mathrm{LiYF}_{4}$ and $\mathrm{LiYbF}_{4}$. Phys. Rev. B 2001, 64, 024304.

(24) Sen, A.; Chaplot, S. L.; Mittal, R. Effects of pressure and temperature on the vibronic as well as the thermodynamic properties of $\mathrm{LiYF}_{4}$ and $\mathrm{LiYbF}_{4}$. J. Phys.: Condens. Matter 2002, 14, 975-986.

(25) Sen, A.; Chaplot, S. L.; Mittal, R. Molecular dynamics simulation of pressure-induced phase transitions in $\mathrm{LiYF}_{4}$ and $\mathrm{LiYbF}_{4}$. Phys. Rev. B 2003, 68, 134105.

(26) Sen, A.; Chaplot, S.; Mittal, R. Lattice vibrations in high-pressure phases of LiYF 4 . Physica B: Condensed Matter 2005, 363, 213 - 224.

(27) Errandonea, D.; Manjon, F.; Somayazulu, M.; Hüsermann, D. Effects of pressure on the local atomic structure of $\mathrm{CaWO}_{4}$ and $\mathrm{YLiF}_{4}$ : mechanism of the scheelite to wolframite and scheelite to fergusonite transitions. J. Solid State Chem. 2004, 177, 1087-1097.

(28) Bensalah, A.; Guyot, Y.; Brenier, A.; Sato, H.; Fukuda, T.; Boulon, G. Spectroscopic properties of $\mathrm{Yb}^{3+}$ : $\mathrm{LuLiF}_{4}$ crystal grown by the Czochralski method for laser applications and evaluation of quenching processes: a comparison with Yb3+: YLiF4. J. Alloys Compd. 2004, 380, 15-26.

(29) Sugiyama, A.; Katsurayama, M.; Anzais, Y.; Tsuboi, T. Spectroscopic properties of Yb doped YLF grown by a vertical Bridgman method. J. Alloys Compd. 2006, 412, 780-783. 
(30) Wybourne, B. Spectroscopic Properties of Rare Earths; Interscience Publisher, 1965.

(31) Malta, O. The Theory of Vibronic Transitions in Rare Earth Compounds. J. Phys. Chem. Solids 1994, 56, 1053-1062.

(32) Journaux, B.; Daniel, I.; Petitgirard, S.; Cardon, H.; Perrillat, J.; Caracas, R.; Mezouar, M. Salt partitioning between water and high-pressure ices. Implication for the dynamics and habitability of icy moons and water-rich planetary bodies. Earth Planet. Sci. Lett. 2017, 463, 36-47.

(33) Journaux, B.; Daniel, I.; Caracas, R.; Montagnac, G.; Cardon, H. Influence of $\mathrm{NaCl}$ on ice VI and ice VII melting curves up to $6 \mathrm{GPa}$, implications for large icy moons. Icarus 2013, 226, 355-363.

(34) Kantor, I.; Prakappenka, V.; Kantor, A.; Dera, P.; Kurnosov, A.; Sinogeikin, S.; Dubrovinskaia, N.; Dubrovinsky, L. BX90: A new diamond anvil cell design for Xray diffraction and optical measurements. Rev. Sci. Instrum. 2012, 85, 125102.

(35) Noguchi, N.; Okuchi, T. A Peltier cooling diamond anvil cell for low-temperature Raman spectroscopic measurements. Rev. Sci. Instrum. 2016, 87, 125107. 


\section{Graphical TOC Entry}

Some journals require a graphical entry for the Table of Contents. This should be laid out "print ready" so that the sizing of the text is correct.

Inside the tocentry environment, the font used is Helvetica 8 pt, as required by Journal of the American Chemical Society.

The surrounding frame is $9 \mathrm{~cm}$ by $3.5 \mathrm{~cm}$, which is the maximum permitted for Journal of the American Chemical Society graphical table of content entries. The box will not resize if the content is too big: instead it will overflow the edge of the box.

This box and the associated title will always be printed on a separate page at the end of the document. 